\title{
CORRIGENDUM
}

\section{Oxidative stress induces mitochondrial dysfunction in a subset of autistic lymphoblastoid cell lines}

\author{
S Rose, RE Frye, J Slattery, R Wynne, M Tippett, S Melnyk and SJ James
}

Translational Psychiatry (2015) 5, e526; doi:10.1038/tp.2015.29; published online 10 March 2015

\author{
Correction to: Translational Psychiatry (2014) 4, e377; doi:10.1038/ \\ tp.2014.15; published online 1 April 2014
}

We would like to clarify the motivation and intent of two different publications with similar purpose, methodology and conclusions. In the journal PLoS One on Jan 8th 2014 we published a paper entitled 'Oxidative stress induces mitochondrial dysfunction in a subset of autism lymphoblastoid cell lines in a well-matched case control cohort.. ${ }^{1}$ This paper described a new type of mitochondrial dysfunction that was found in the autistic but not the control cell lines in which physiological levels of reactive oxygen species resulted in a significant reduction in mitochondrial Reserve Capacity in a subset of cell lines derived from children with autism spectrum disorder. In our previous research we have always used adult cell lines as control cell lines when examining cell lines derived from children with autism because control cell lines from children are much more difficult to obtain. ${ }^{2}$ Because of the novelty of our research, during its development, multiple colleagues made the important observation that the use of adult control cell lines would be suboptimal. Specifically, it would leave open the question whether the changes could be due to differences in the ages of the autistic and control participants who donated the cells. Based on this suggestion, we performed the experiment published in the PLoS One paper as was expressed in the title - '... a well-matched case control cohort.' The PLoS One paper also presented some very detailed analysis of specific proteins that could account for the effect found. In particular, Western Blots demonstrated that Uncoupling Protein 2, a key protein in the regulation of oxidative stress at the inner mitochondrial membrane, was significantly different between the two subsets of autistic cell lines.
Like all scientific research, the experiment published in PLoS One, required validation and elaboration. The PLoS One paper still left open the question whether adult cell lines could be used as a valid control when examining autistic cell lines. Establishing the use of adult control cell lines as a useful comparison group is very important as it validates our previous work ${ }^{2}$ and the work of others who commonly use adult control cell lines. To this end we published a paper in Translational Psychiatry entitled 'Oxidative stress induces mitochondrial dysfunction in a subset of autistic lymphoblastoid cell lines' on February 2nd 2014 which asks a similar question as the PLOS One paper and confirmed our previous findings using similar (but not the same) methodology but with adult control cell lines. ${ }^{3}$ The significance and novelty of the Translational Psychiatry paper were three-fold. First, the Translational Psychiatry paper confirmed the results seen in the PLoS One paper; second, it demonstrated that the use of adult control cell lines is valid, at least for mitochondrial studies, when examining cell lines derived from autistic children; and, third, we demonstrated the protective effect of $\mathrm{N}$-acetylcysteine on the subset of autistic cell lines that were found to be vulnerable to oxidative stress.

For clarity, we would like to take this opportunity to point out the differences between the two papers. Table 1 outlines the 12 differences in the experiments reported in the two papers. (1) As previously mentioned, the PLoS One article used carefully agematched childhood control cell lines while the Translational Psychiatry article used adult control cell lines. $(2,3)$ The two papers used different numbers of both autistic and control cell lines. (4) The design of the Seahorse cartridges used in the Seahorse Extracellular Flux 96 Analyzer (Seahorse Bioscience, North Billerica, MA, USA) was different. (5) Because of the differences in the

Table 1. Difference between two publications on oxidative stress inducing mitochondrial dysfunction in autistic lymphoblastoid cell lines

\begin{tabular}{llll}
\hline ref & PLoS One & Translational Psychiatry \\
\hline 1 & Controls Cell Lines & Carefully Matched on Age & Adults not Matched on Age \\
2 & Number of Autistic Cell Lines & 25 Cell Lines & 22 Cell Lines \\
3 & Number of Control Cell Lines & 13 Cell Lines & 14 Cell Lines \\
4 & Seahorse Cartridge & 4-port cartridge & 2-port cartridge \\
5 & Statistical Analysis & Individual Well Data & Average Well Data \\
6 & Clustering Variables & Baseline Reserve Capacity and Change in Reserve & Change in Proton Leak and Change in \\
& & Capacity & ATP-Linked Respiration \\
7 & Pretreatment Agent & Genipin & N-acetylcysteine \\
8 & Cellular Proteins Examined by & UCP2 & None \\
& Western Blot & ND1, ND4, Cyt B & None \\
9 & Mitochondrial Genes & GSH, GSG/GSSG, Cysteine/Cystine, NADH/NAD+, & GSH, GSSG, GSG/GSSG \\
10 & Intracellular Redox & 3-Nitrotyrosin, CellROX Green & Not Done \\
11 & Mitochondrial Redox & MitoSOX & Not Done \\
12 & Mitochondrial Membrane Potential & JC1 &
\end{tabular}


cartridges we could only use individual well data in the PLoS One experiment and needed to use data averaged across wells in the Translational Psychiatry paper. (6) The variables used to perform the cluster analysis were different. (7) The agents used to pretreat the cell lines for the follow-up experiment were different.

(8) Cellular proteins were only examined in the PLoS One paper. (9) Mitochondrial genes were only examined in the PLoS One paper. (10) A more extensive set of intracellular redox markers were examined in the PLoS One paper. (11) Mitochondrial Redox was only examined in the PLoS One paper. (12) Mitochondrial membrane potential was only examined in the PLOS One article.

Since these two papers had a generally similar purpose, methodology and conclusions, some have noted the similarity in the text in the Introduction, Methods and Conclusion with the same wording in several places as well as the use of the same methodology figure (Figure 1 in both papers), representing concepts that are common to our work on mitochondrial function in autism. The authors sincerely apologize for the duplicated text that appears in the Introduction, Methods, Discussion, and Conclusion.

\section{REFERENCES}

1 Rose S, Frye RE, Slattery J, Wynne R, Tippett M, Pavliv $\mathrm{O}$ et al. Oxidative stress induces mitochondrial dysfunction in a subset of autism lymphoblastoid cell lines in a well-matched case control cohort. PLoS One 2014; 9: e85436.

2 James SJ, Rose S, Melnyk S, Jernigan S, Blossom S, Pavliv O et al. Cellular and mitochondrial glutathione redox imbalance in lymphoblastoid cells derived from children with autism. FASEB J 2009; 23: 2374-2383.

3 Rose S, Frye RE, Slattery J, Wynne R, Tippett M, Melnyk S et al. Oxidative stress induces mitochondrial dysfunction in a subset of autistic lymphoblastoid cell lines. Transl Psychiatry 2014; 4: e377. 